\title{
An Unusual Case of Myelopathy: Surfer's Myelopathy
}

\author{
P.P.S. Dhaliwal, A. Cenic, M. Eesa, S. du Plessis
}

Can J Neurol Sci. 2011; 38: 354-356

Myelopathy is a term that has been used to describe a condition that reflects impairment in spinal cord function ${ }^{1}$. It is typically characterized by loss of bilateral motor function and altered sensation below the level of the lesion. There is often concomitant autonomic impairment manifested as urinary or bowel dysfunction. Causes for myelopathy can be discussed based on the temporal evolution of dysfunction as well as the extent of spinal cord involvement. Etiologically, one can broadly categorize acute myelopathy into traumatic or non-traumatic causes $^{2,3}$, (see Table).

In developed countries, athletic injury is the third most common cause of traumatic spinal cord injury and may represent up to $20 \%$ of new cases ${ }^{4}$. Though preventative strategies have been developed, the burden of injury and disability remains significant. Certain sports ${ }^{5}$ (including diving, hockey, football, skiing, wrestling) are recognized as higher risk activities in the context of spinal cord injury. Though most spinal cord injuries associated with sports and recreation are related to trauma, here we describe a case of non-traumatic acute spinal cord injury associated with surfing.

\section{Case Presentation}

\section{Clinical Scenario}

A previously healthy 29 year-old male was on vacation in Hawaii. While taking his first surfing lesson, he noted a spasm in his back while paddling on the surfboard in a prone position. While continuing on with his surfing lesson he noticed progressive weakness in his lower extremities. He made his way to the beach and had great difficulty walking. In the following 30 minutes, he lost complete strength in his lower extremities and developed paresthesias in both of his lower extremities. He was taken urgently to the local hospital for further assessment. The patient denied any blunt trauma during the surfing lesson to his spine. There was no loss of consciousness or head trauma, and no antecedent history of neck or back pain, fever, infections or malignancy. He did however have a remote history of bilateral deep venous thrombosis. He was not on any medications prior to this incident.

\section{Physical Examination}

On initial presentation the patient had stable vital signs. Cranial nerve examination revealed pupils which were equal and reactive to light, normal visual acuity, normal fundoscopy and normal visual fields. Extraocular movements were full and there was no facial asymmetry or sensory changes. There was no tongue deviation and the palate rose symmetrically. Motor examination revealed grade $5 / 5$ strength in the upper extremities
Table: Differential diagnosis of non-traumatic noncompressive acute myelopathy

\begin{tabular}{l|l}
\hline & CAUSES \\
\hline Viral or post-viral & $\begin{array}{l}\text { Enteroviruses, human immunodeficiency } \\
\text { viruses (HIV), human T-lymphotrophic } \\
\text { virust-1 (HTLV-1), Epstein Barr Virus, } \\
\text { (EBV), cytomegalovirus (CMV), varicella } \\
\text { zoster virus (VZV), acute disseminated } \\
\text { encephalomyelitis (ADEM) }\end{array}$ \\
\hline $\begin{array}{l}\text { Vasculitic/multisystem } \\
\text { diseases }\end{array}$ & $\begin{array}{l}\text { Systemic lupus erythematosis, Behcet's } \\
\text { syndrome, Sjogren's syndrome, Wegener's } \\
\text { syndrome, sarcoid }\end{array}$ \\
\hline Demyelinating disease & Multiple sclerosis, neuromyelitis optica \\
\hline Vascular lesions & Arteriovenous malformations, cavernomas \\
\hline $\begin{array}{l}\text { Toxic/metabolic } \\
\text { causes }\end{array}$ & $\begin{array}{l}\text { Vitamin B } \\
\text { injections }\end{array}$ \\
\hline Paraneopiciency, heroin, epidural \\
\hline Electrical injury & \\
\hline
\end{tabular}

bilaterally. In the lower extremities however, there was grade $0 / 5$ strength in all major muscle groups of the lower extremities bilaterally. Sensory examination revealed paresthesias to both lower extremities from the inguinal region distally. Tone and reflexes were normal in the upper extremity but absent in the lower extremities bilaterally. Plantar responses were nondiagnostic. General systems examination was unremarkable.

\section{Investigations}

Laboratory investigations demonstrated normal hemoglobin and white blood cell count. Electrolytes were normal. Magnetic resonance imaging (MRI) of the brain and spinal cord was performed at the time of initial presentation and showed high signal intensity in the lower thoracic cord and conus medullaris on T2 weighted imaging (Figure 1). This was predominantly located within the central portions of the cord and more ventrally

From the Division of Neurosurgery (PPSD, AC, SdP), University of Calgary, Calgary, Alberta, Canada; Department of Radiology (ME), Columbia University, New York, New York, U.S.A.

Received May 19, 2010. Final Revisions Submitted October 12, 2010 Correspondence to: Perry Dhaliwal, Division of Neurosurgery, Foothills Medical Center, 1403 29th Street N.W., Calgary, Alberta, Canada, T2N 4T9. 


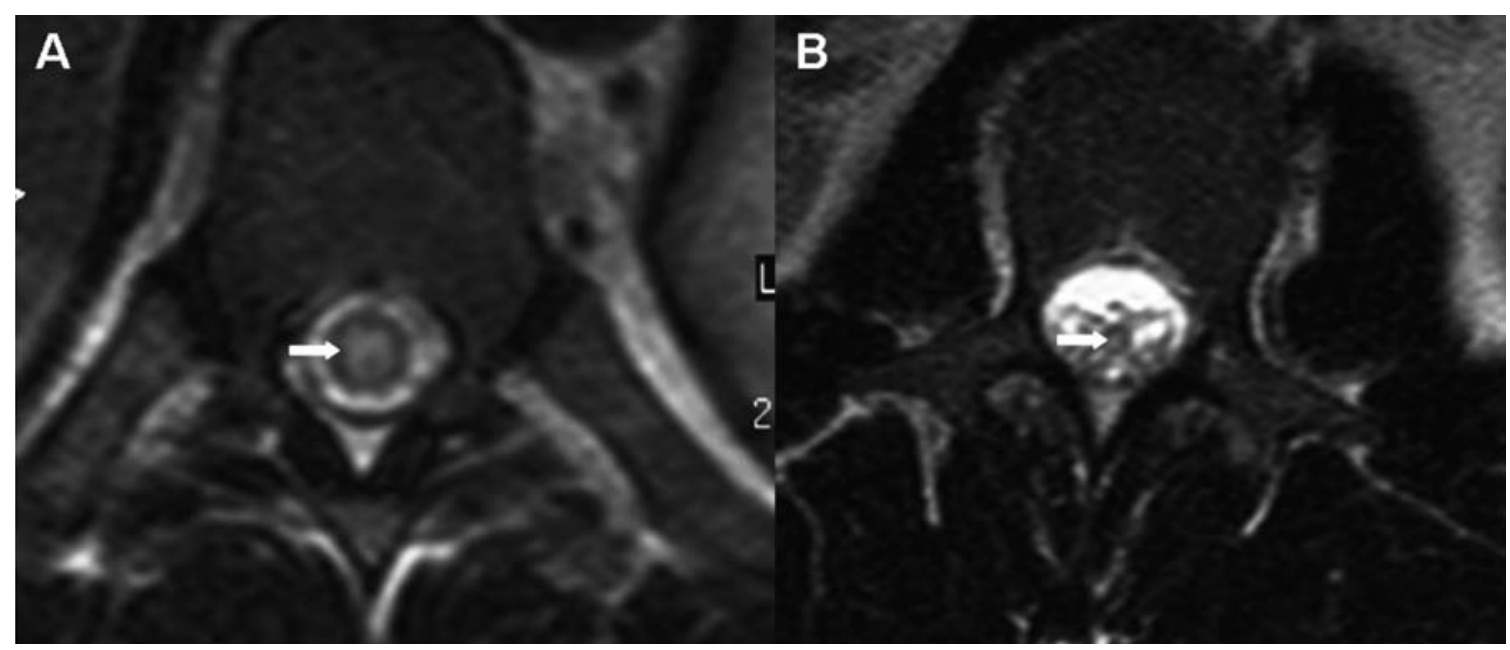

Figure 1: Axial T2 weighted MR sections of the lower thoracic cord (A) and conus (B) at presentation shows increased signal (white arrows) within the central portions of the cord and conus.

in the distribution of the anterior spinal artery territory (Figure 2). There was no abnormal enhancement of the spinal cord or epidural space at the time of initial presentation.

Upon initial presentation, the patient was started on high dose steroids for 24 hours. Over the ensuing hospital stay, the patient gradually regained motor function in his lower extremities. Upon neurological assessment one week after initial presentation, the patient was noted to have grade $3 / 5$ strength in all major muscle groups of his lower extremities bilaterally. The patient was then transferred to Calgary, Alberta (Canada) for ongoing management and rehabilitation. Repeat MRI of the spine showed partial resolution of the high $\mathrm{T} 2$ signal change in the spinal cord. There was also enhancement around the conus and the ventral roots of the cauda equina (see Figure 3). This was favored to represent a secondary inflammatory reaction in response to the initial ischemic injury corresponding to the anterior spinal artery territory. Nerve conduction studies were also performed which did not demonstrate any nerve conduction abnormalities.

Overall, this patient's clinical picture appeared to be consistent with myelopathy secondary to an ischemic insult.

\section{Discussion}

Thompson et $\mathrm{al}^{6}$ first described a series of nine patients with a very similar presentation to that of our patient. These patients were also inexperienced first-time surfers, who had acute onset of symmetric leg weakness soon after beginning surfing. They also had a variable constellation of symptoms including back pain, hyperesthesia, hypalgesia and urinary retention. Magnetic resonance investigations performed on these patients demonstrated hyperintense signal changes in the lower thoracic spinal cord extending to the level of the conus medullaris. There was no evidence of underlying thoracic or upper lumbar spondylosis in these patients. Of those patients with serial MRI investigations, two had rostral progression of the signal change while it improved in one patient. Though there was limited follow-up, only one of nine patients was paraplegic, four had residual weakness and three patients continued to have urinary retention at the time of discharge from hospital. A similar case was reported by Aviles-Hernandez et $\mathrm{al}^{7}$ in 2007 involving a novice surfer with sudden onset back pain which progressed to paresthesias and weakness in the lower extremities following a surfing lesson.

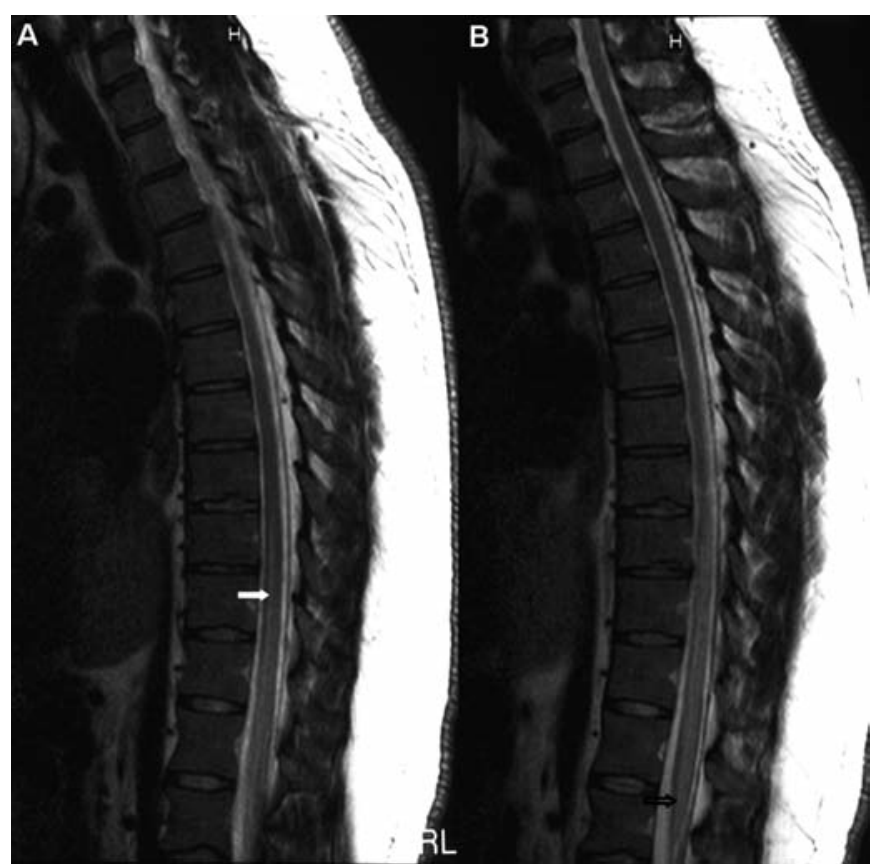

Figure 2: Sagittal T2 weighted MRI showing the extent of signal change predominantly in the anterior regions (white arrow in A) of the lower thoracic cord with extension into the conus (open black arrow in B). 


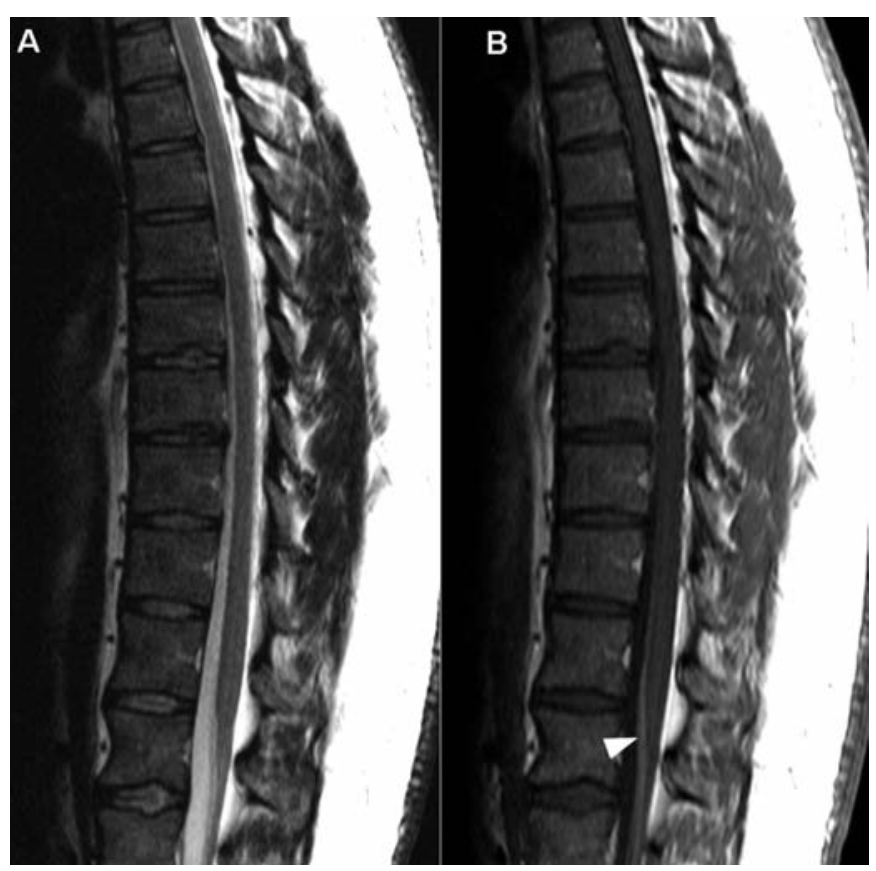

Figure 3: Follow-up MRI showing partial resolution of the T2 signal change (A) with peripheral enhancement of the lower cord and conus on post gadolinium MR scans (arrowhead in B).

Despite the clear pattern of neurological injury on clinical examination and radiological imaging in this group of patients, the pathophysiology remains unclear. An ischemic injury is postulated to be the underlying cause, though the exact mechanism is uncertain. Thompson et al had postulated that hyperextension of the spine while lying prone on the surfboard may have led to vascular compromise hence and spinal cord ischemia ${ }^{6}$. Other possibilities include: vasospasm of the artery of Adamkiewicz, tearing of perforating vessels, and transient hypoperfusion in the spinal cord in the lower thoracic water-shed regions of the spinal cord. It is postulated that fibrocartilaginous emboli from a disk herniation may also cause spinal cord ischemia by traveling through the epidural venous plexus or via the central arteries ${ }^{8}$. However, the exact mechanism is not known ${ }^{8}$. Though arterial injury is thought to be the underlying cause, venous congestion of the spinal cord may also be a possibility as venous outflow is obstructed as patients lie prone on the surfboard.

Similar to these series of patients, our patient had acute onset weakness of the lower extremities with sensory disturbance and urinary retention. Over the course of days, he was noted to have resolution of the signal change in the spinal cord and concomitant improvement of motor function. Both clinical and radiological findings were felt to be consistent with a transient ischemic injury to the spinal cord. Interestingly, the patient did have a remote history of deep vein thrombosis which was felt to be secondary to androgen use. Previous hematological investigations were not available for review but there was no family history of venous thrombosis reported by the patient or family.

\section{SUMMARY}

First reported in 2004, surfer's myelopathy is a form of nontraumatic myelopathy that occurs in novice surfers usually during the first attempt at surfing. Patients usually have a sudden onset of back pain followed by symmetric leg weakness and variable sensory changes in the lower extremities. The MRI investigations demonstrate increased signal intensity on $\mathrm{T} 2$ images of the lower thoracic spinal cord extending to the conus medullaris which tends to resolve over time on repeat imaging. Current experience with surfer's myelopathy would suggest that most patients show varying degrees of improvement in motor function over time, however, long-term follow-up is required. Though uncommon in Canada, this clinical presentation is unusual in that an athletic activity may result in a non-traumatic spinal cord injury; hence, this case report serves as a reminder to consider all possible etiologies for acute spinal cord injury.

\section{REFERENCES}

1. Scotti G, Gerevini S. Diagnosis and differenital diagnosis of acute transverse myelopathy. The role of neuroradiological investigation and review of the literature. Neurol Sci. 2001;22: S69-73.

2. Ghezzi A, Baldini SM, Zaffaroni M. Differential diagnosis of acute myelopathies. Neurol Sci. 2001;22:S60-4.

3. Harzheim M, Schlegel U, Urbach H, Klockgether T, Schmidt S. Discriminatory features of acute transverse myelitis: a retrospective analysis of 45 patients. J Neurol Sci. 2004;217: 217-23.

4. Tator $\mathrm{CH}$. Recognition and management of spinal cord injuries in sports and recreation. Neurol Clin. 2008;26(1)79-88.

5. Boden BP, Jarvis CG. Spinal injuries in sports. Neurol Clin. 2008; 26:63-78.

6. Thompson T, Pearce J, Chang G. Madamba J. Surfer's myelopathy. Spine. 2004;29(16):E353-6.

7. Aviles-Hernandez I, Garcia-Zozaya I, DeVillasante JM. Nontraumatic myelopathy associated with surfing. J Spinal Cord Med. 2007;30:288-93.

8. Han, J, Massagli T, Jaffe K. Fibrocartilaginous embolism- an uncommon cause of spinal cord infarction: a case report and review of the literature. Arch Phys Med Rehabil. 2004;85:153-7. 